\title{
PROBLEMS CAUSED BY THE PANDEMIC IN PUBLIC PROCUREMENT OF GEORGIA
}

\section{GVANTSA SISOSHVILI}

\section{PhD student}

Ivane Javakhishvili Tbilisi State University, Georgia

gsisoshvili@gmail.com

Abstract. The spread of the new coronavirus in the world, including in Georgia, had a significant negative impact on both public and private organizations, which has affected the public procurement process in the country. Georgia has spent a large amount of money to deal with the virus. As a result, administrative costs have been reduced in order to finance the 2020 state budget deficit - 57 million GEL for labor costs and 107 million GEL for goods and services. It should be noted that 195 million GEL is used on arranging quarantine spaces and other additional health care costs while 1.31 billion GEL is provided for goods and services. During the pandemic, demand from the state increased significantly in the health sector, while declining in other areas, many planned purchases were canceled, tenders were not held, were suspended or their announcement was delayed. This has affected companies whose major activities include state orders. Also rising inflation and currency devaluation have made goods and services more expensive. Consequently, complex problems arose for the entities participating in public procurement, namely:

- From the moment the first case of the virus was reported in Georgia, it became mandatory for public and private organizations to follow the recommendations of the World Health Organization and the National Center for Disease Control and Public Health. So, large amount of money was spent on hygiene, disinfection and other means of protecting against the virus to ensure the safety of customers and employees.

- Some public and private organizations continued to work remotely, for which they incurred additional costs for equipping employees at home (with computer or other technological resources). Other organizations, which were not prohibited the operation, funded the transportation of staff to the workplace, because of emergency was declared and public transport was restricted.

- In order to free up financial resources, procurement organizations have restricted the announcement of planned tenders. Even the winner had already been identified or the bidder had been invited to sign the contract. At the same time, according to the Unified Public Procurement Electronic System a number of tenders have been suspended, including largescale infrastructure projects, most of which were in the selection stage or successful bidder was revealed and invited to sign the contract.

- Due to the global pandemic, most companies failed to meet their contractual obligations on time. Contract authorities change the terms of the contract, including increased delivery time and services. In the case of infrastructure projects, based on the decree of the Government of Georgia, contract authorities were given the right to increase the contract value by $10 \%$ $-20 \%$ in order to finance the increase of supplier costs on construction materials

- Amendments were made to the legislation governing public procurement. For example, Procurement of goods and services under the many of CPV codes were restricted to contract authorities without the consent of the Government of Georgia. In the original version of the resolution, only 30 out of 200 codes were restricted, and from April 1, 2020, the number of restricted CPV codes has increased to 160. There are number of classified goods and services, whose purchase does not require government consent: agricultural and food products, outerwear, pharmaceutical products, miscellaneous transport equipment and spare parts, Works for complete or part construction and civil engineering work, laboratory services, refuse and waste related services. Because agreement with the government and electronic procedures takes a long time, procurement organizations were forced to purchase goods and services through a simplified procurement in the case of urgent necessity. This means that in accordance with the law, the contract authorities directly signed a contract with the company and after agreed with the State Procurement Agency.

Therefore, it is necessary to analyze the additional costs incurred in the field of public procurement due to the coronavirus to make predictions about the procurement process for the next year.

KEYWORDS: PUBLIC PROCUREMENT, PUBLIC ORGANIZATION (CONTRACT AUTHORITIES), PRIVATE ORGANIZATION (SUPPLIER), GOODS AND SERVICES.

For citation: Sisoshvili, G., (2020). Problems caused by the Pandemic in Public Procurement of Georgia. Globalization and Business, 10. 278-286. (In Georgian) https://doi.org/10.35945/gb.2020.10.038 


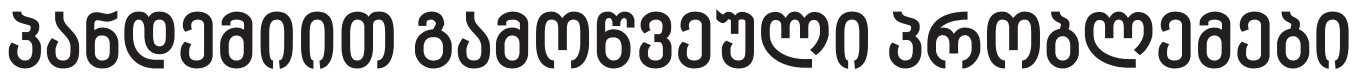

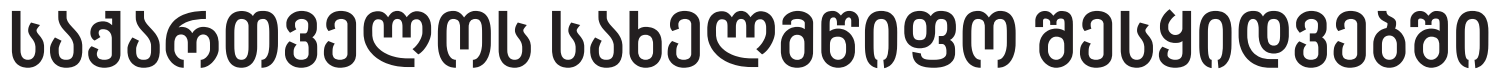

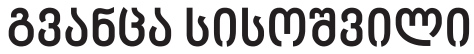

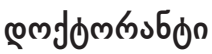

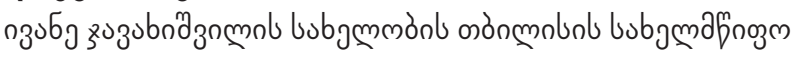

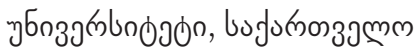 \\ gsisoshvili@gmail.com
}

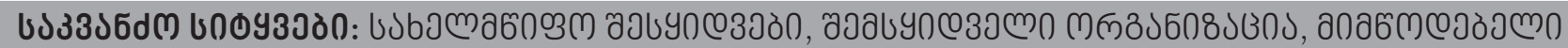

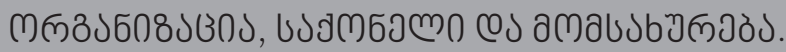

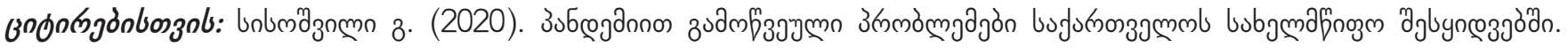

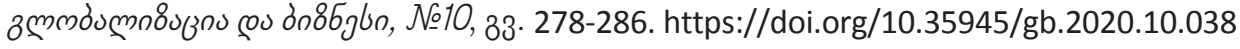

\section{ฆวเธз১ल0}

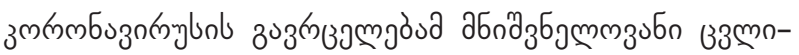

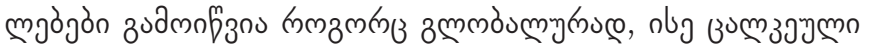

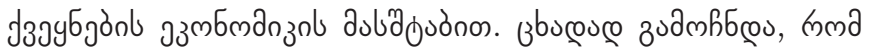

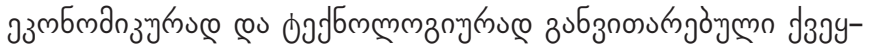

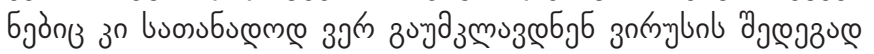

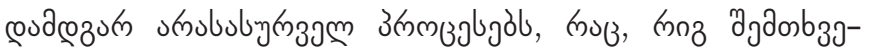

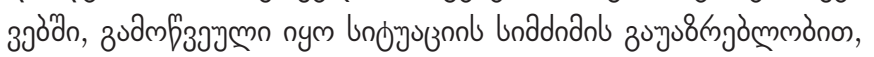

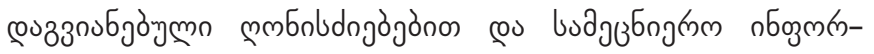

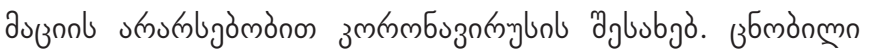

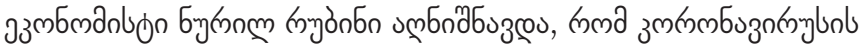

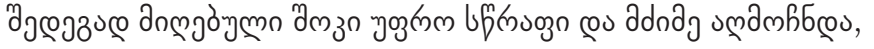

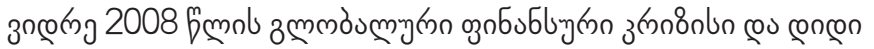

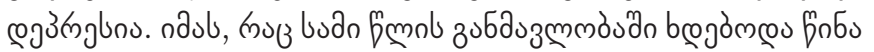

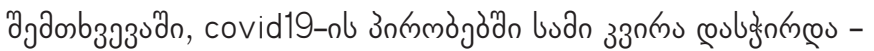

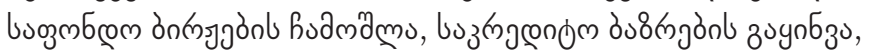

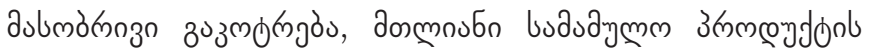

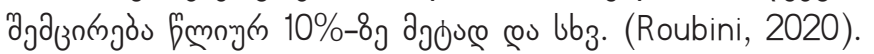

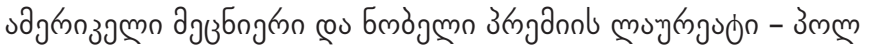

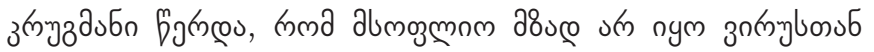

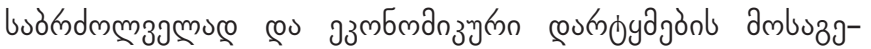

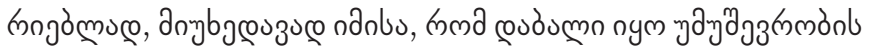

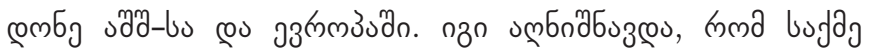

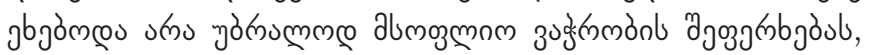

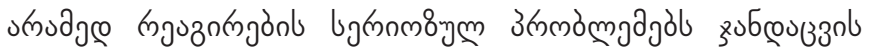

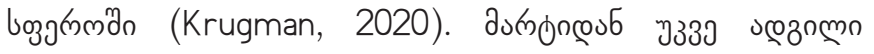

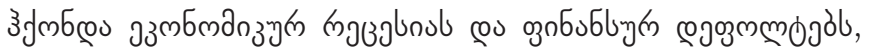

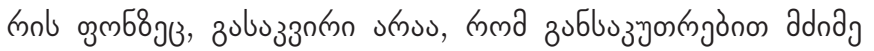

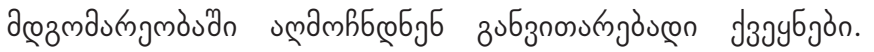

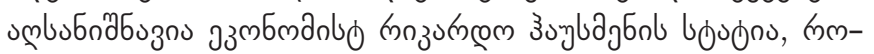

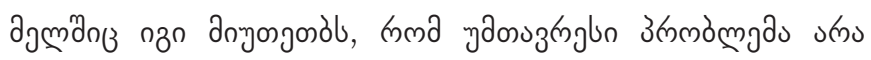

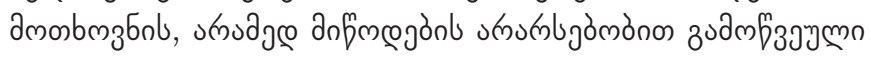

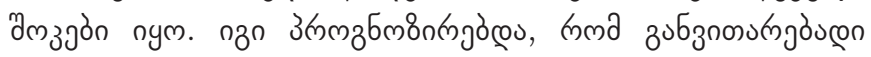

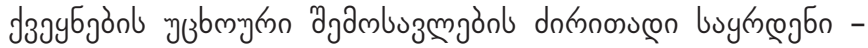

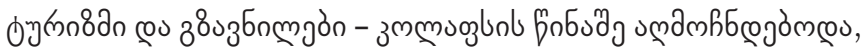

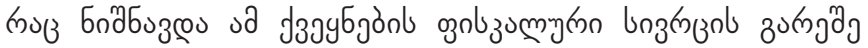
podm3jöbl (Hausmann, 2020).

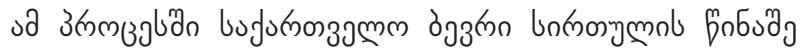

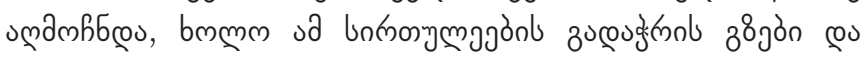

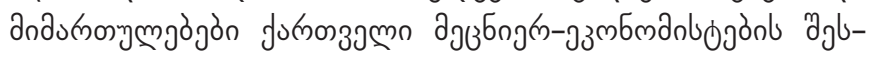

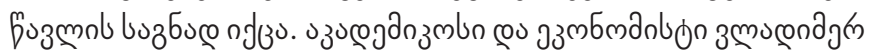

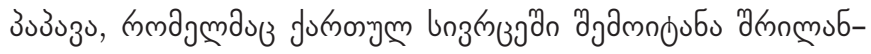

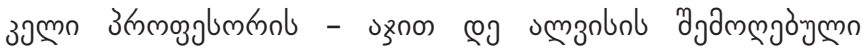

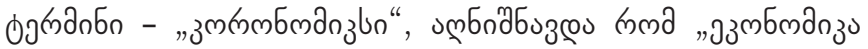

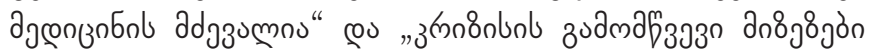

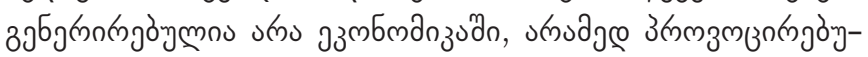

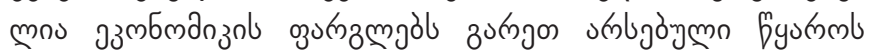

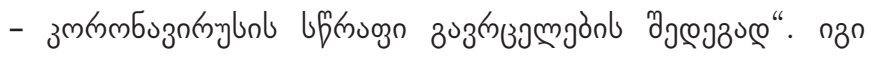

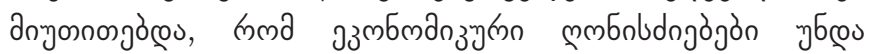

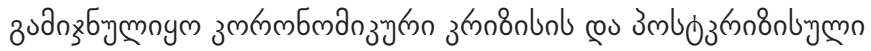

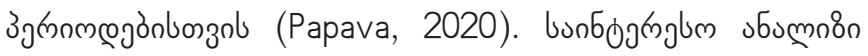

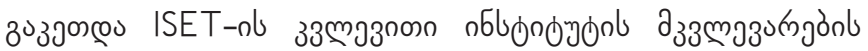

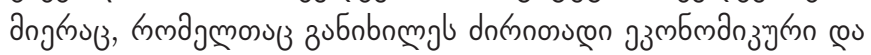

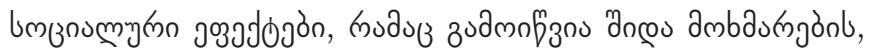
дn œu n

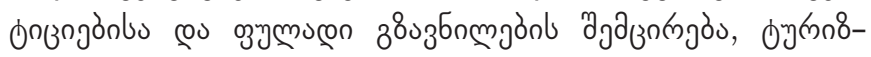

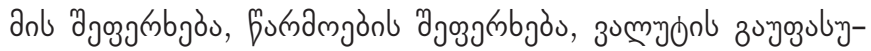
ngoos pos bbz. (Babichi, Keshelava D, Mzhavanadze, 2020).

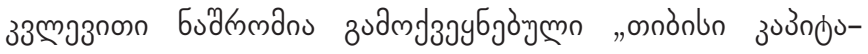

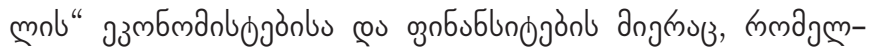

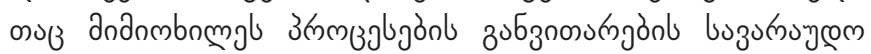




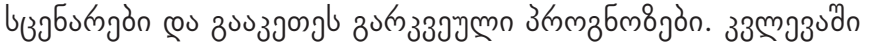

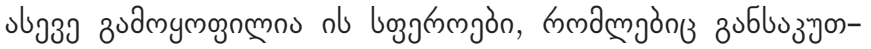

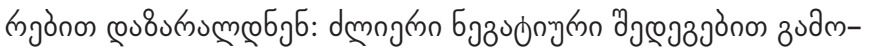
n пһ

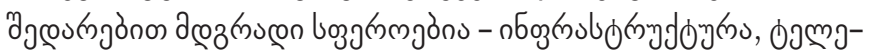

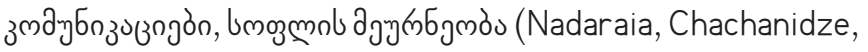
Bluashvili, Kordzaia, 2020).

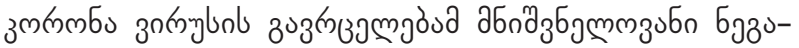

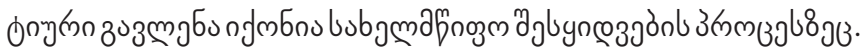

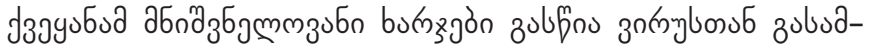

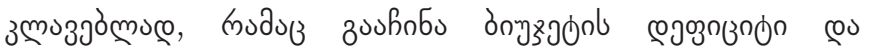

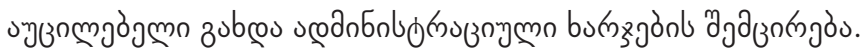

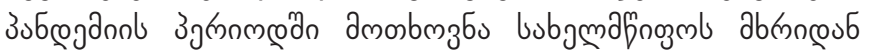

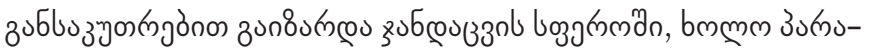

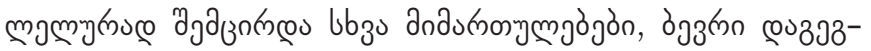

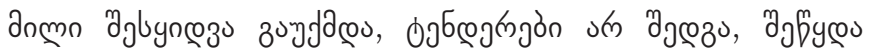

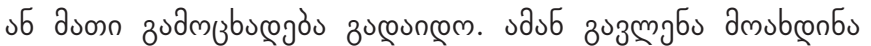

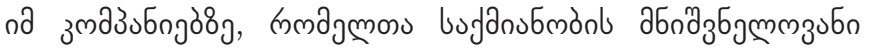

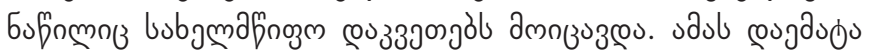

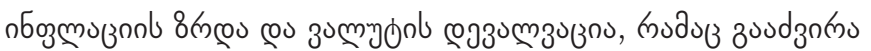

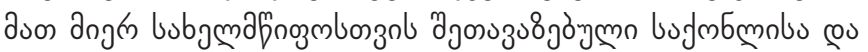

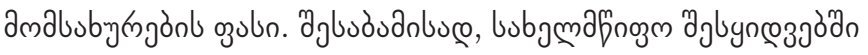

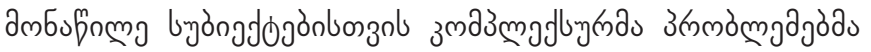

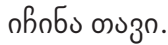

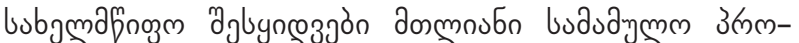

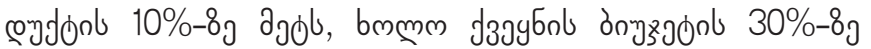

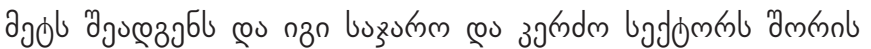

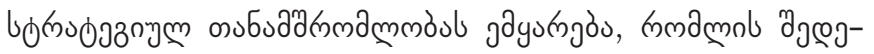

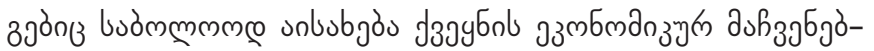

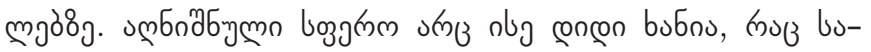

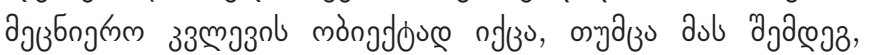

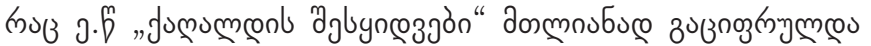

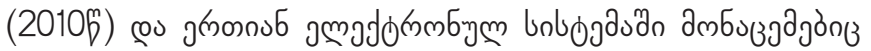

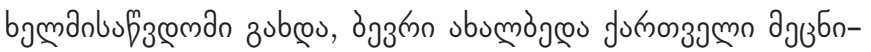

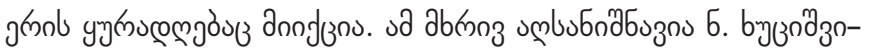

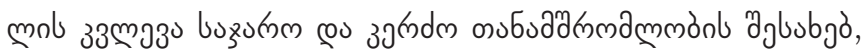

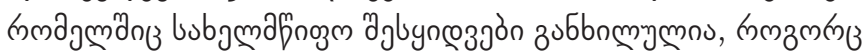

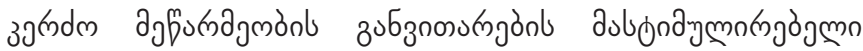

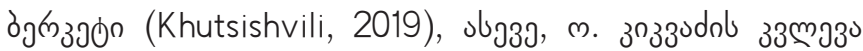

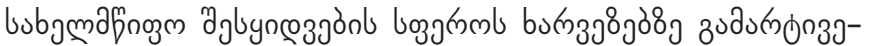

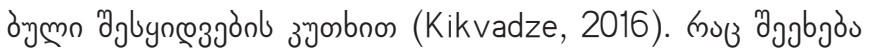

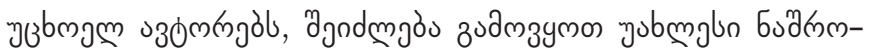

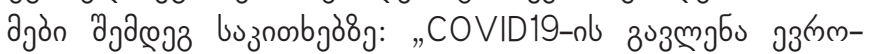
з०3

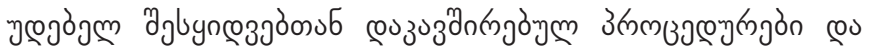

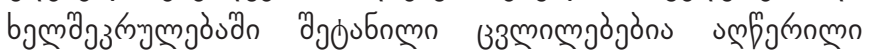

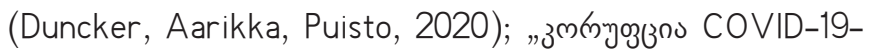

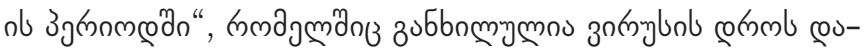

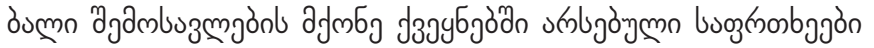

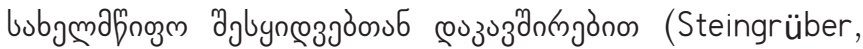

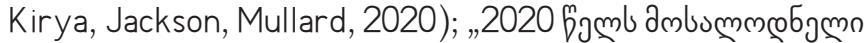

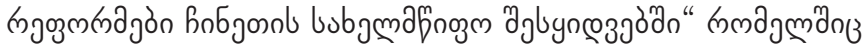

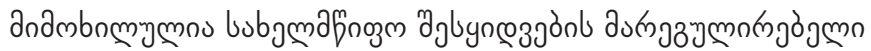

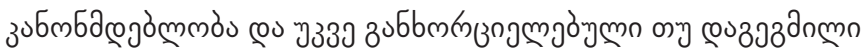

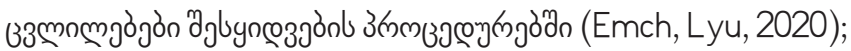

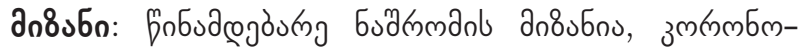

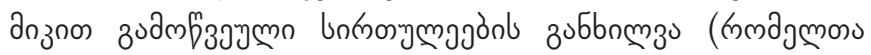

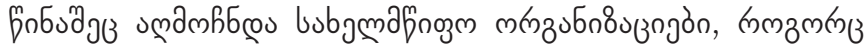

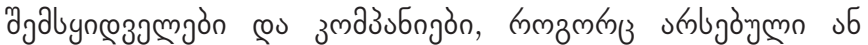

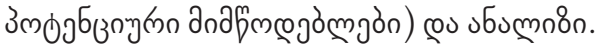

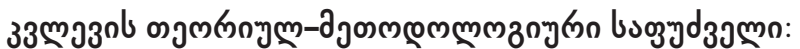

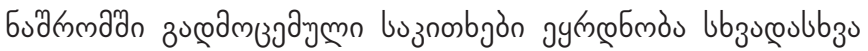

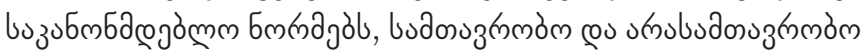

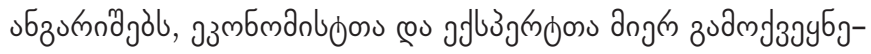

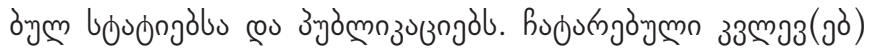

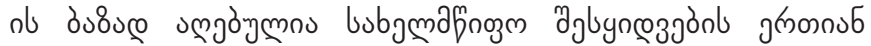

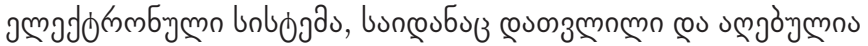

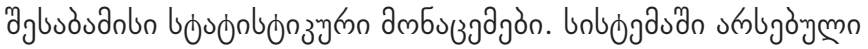

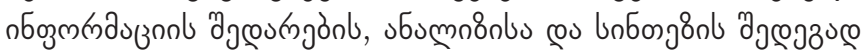

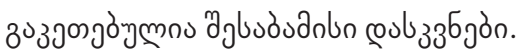

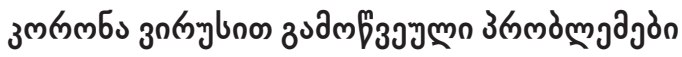

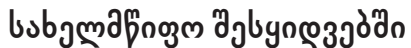

bufunosзs

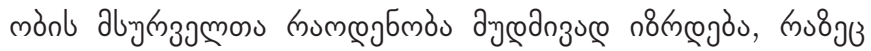

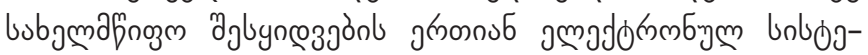

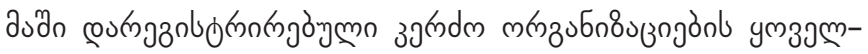

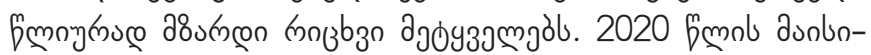

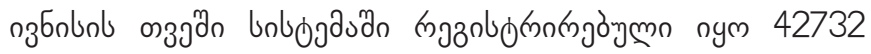

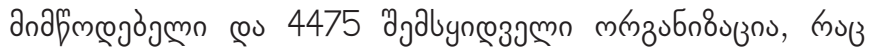

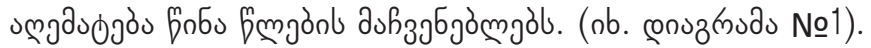
(SPA, Annual report 2019)

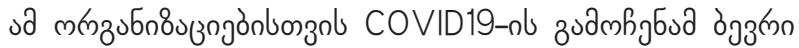

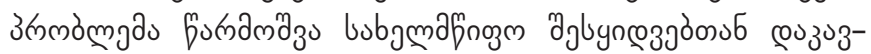

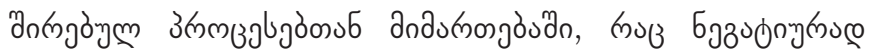

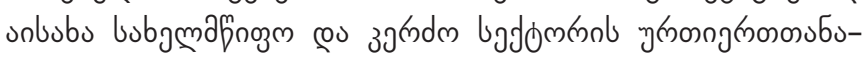

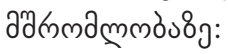

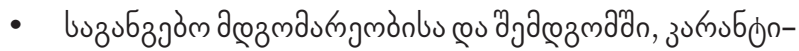

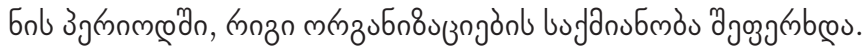

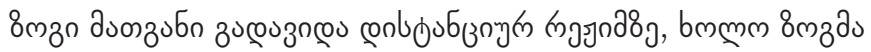

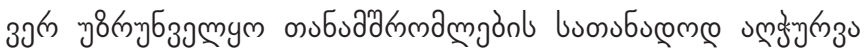

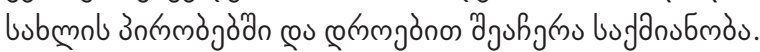

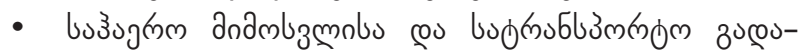

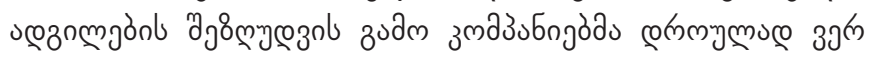

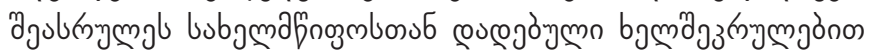

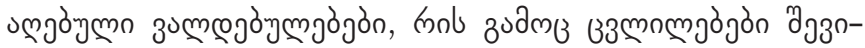
एu bygmaj

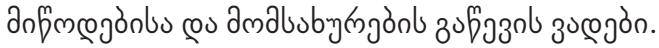

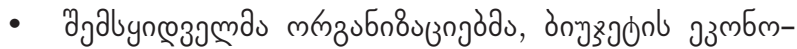




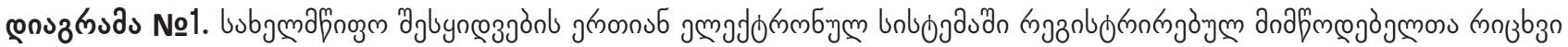
fmegònt dnbgœznos.

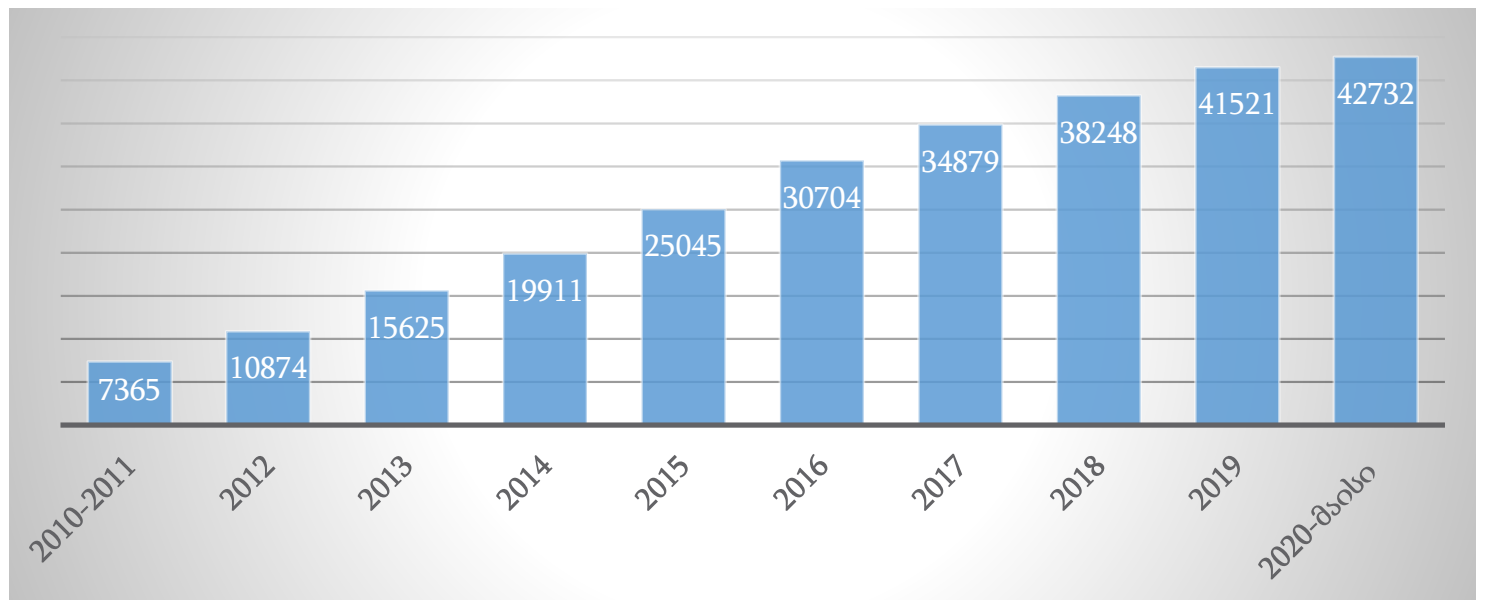

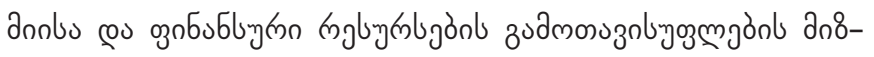

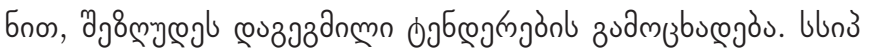

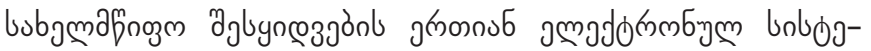

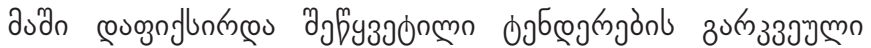

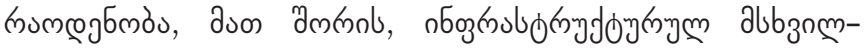

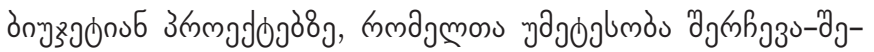

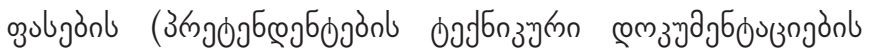

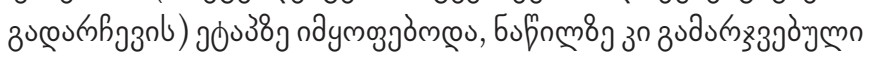

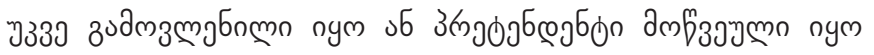

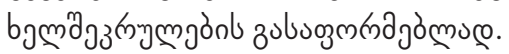

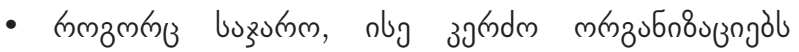

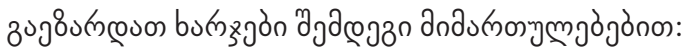

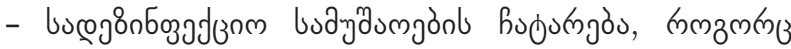

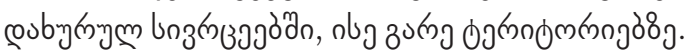

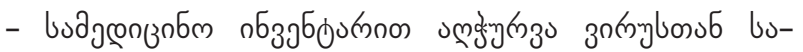

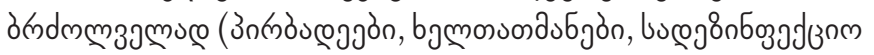

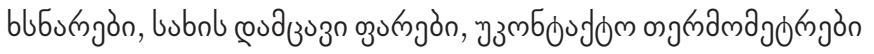
cou bbs.).

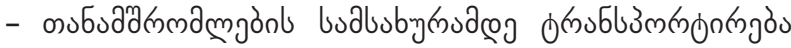

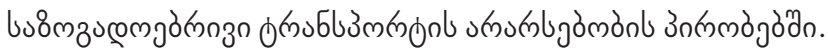

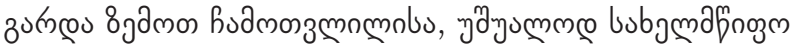

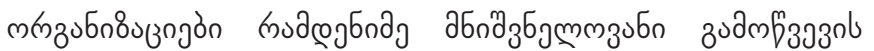

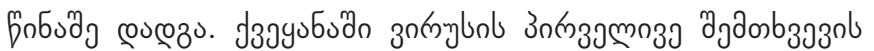

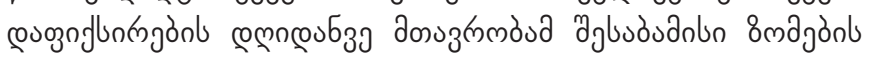

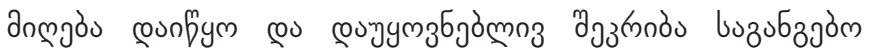

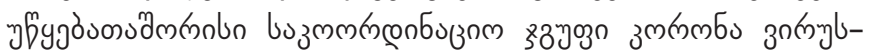

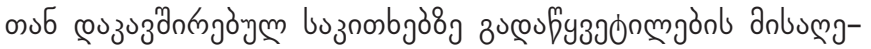

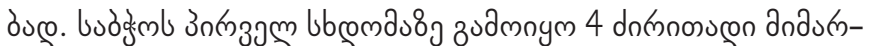

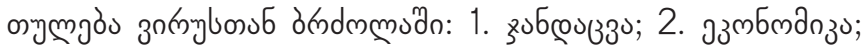

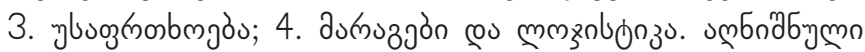

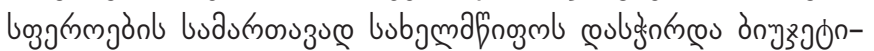

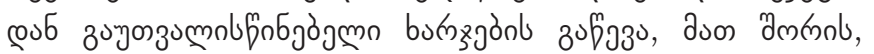

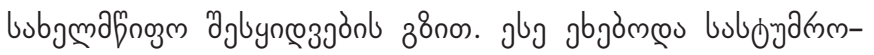

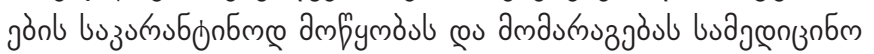

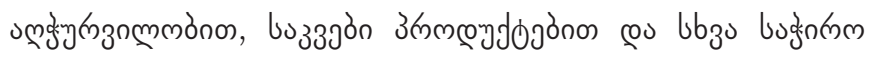

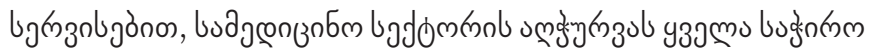

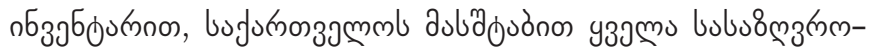

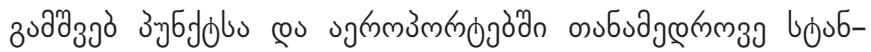

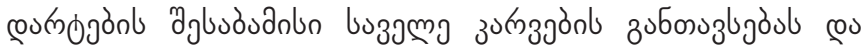

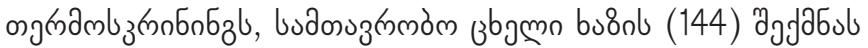
cou bb3. (Rep. of Gov. 2020).

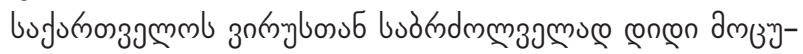

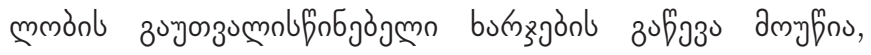

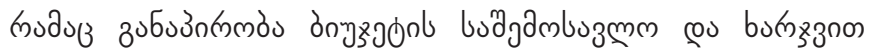

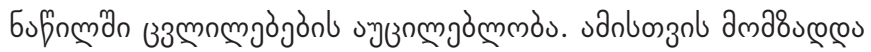

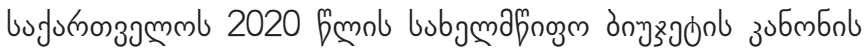

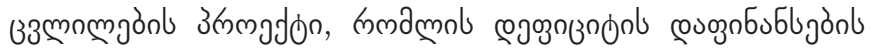

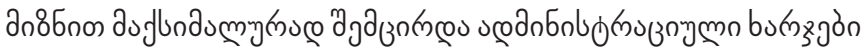

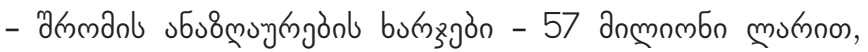

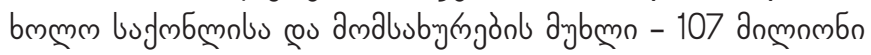

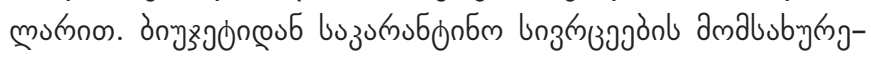

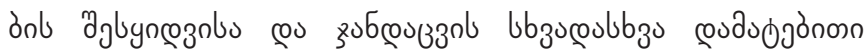

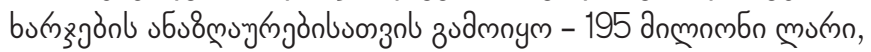

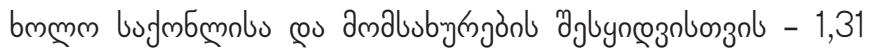

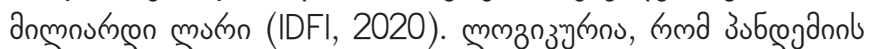

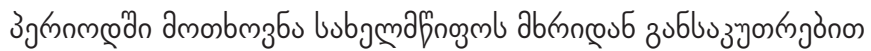

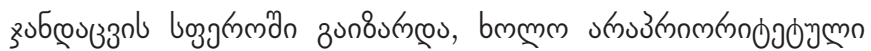

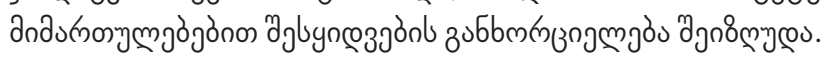

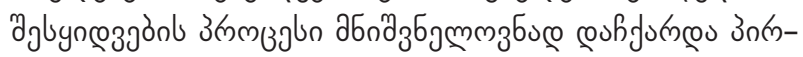

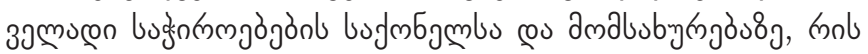

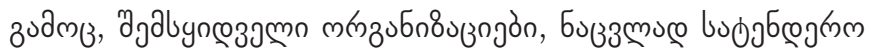

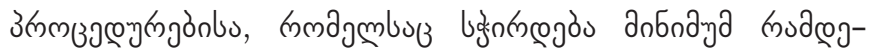

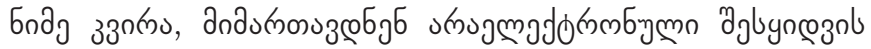

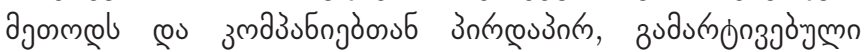

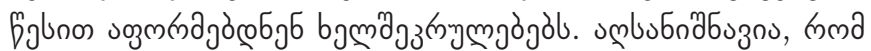

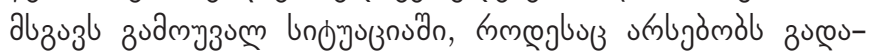

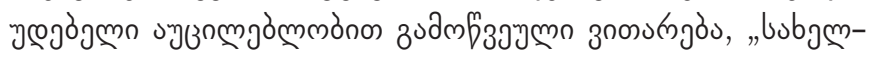

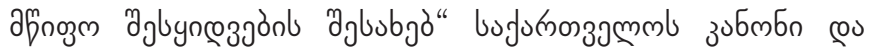




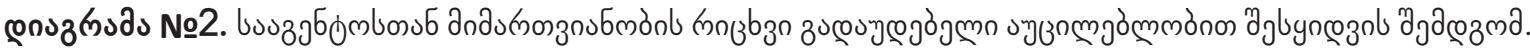

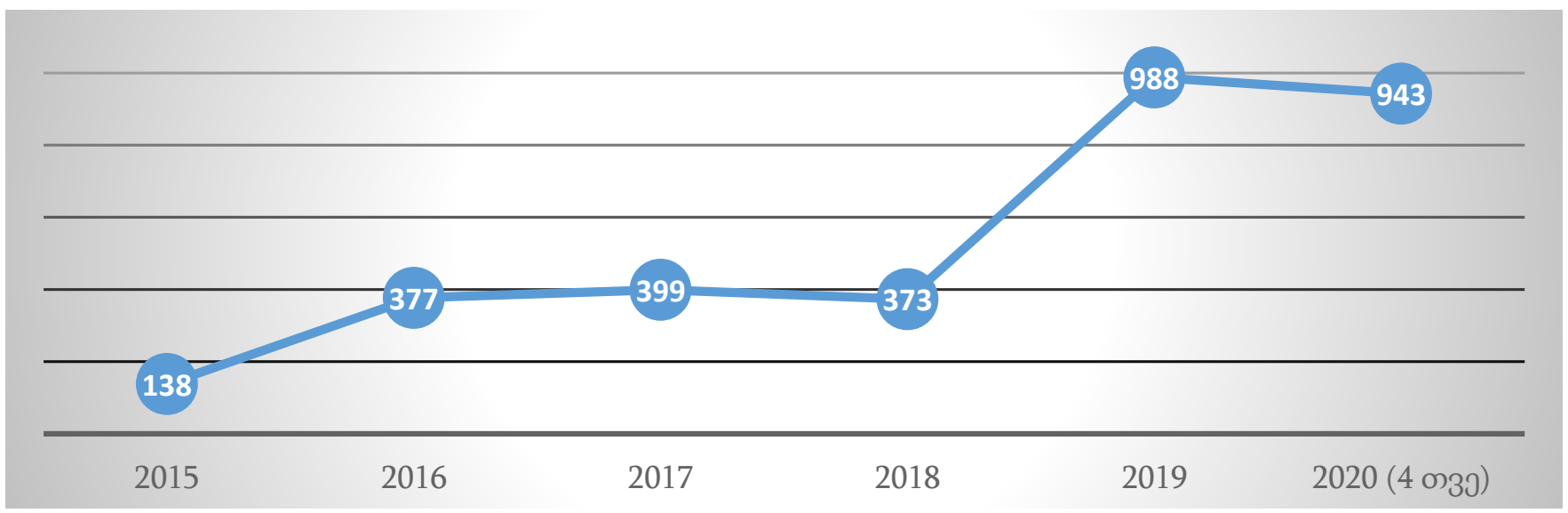

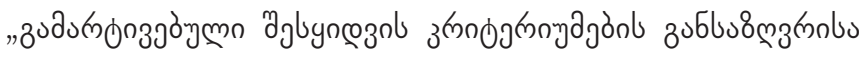

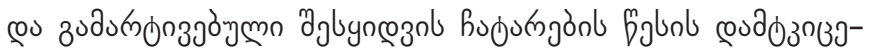

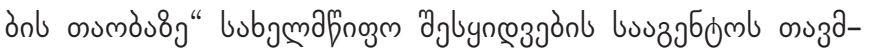

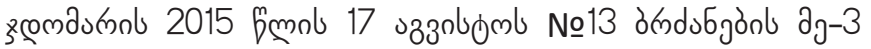

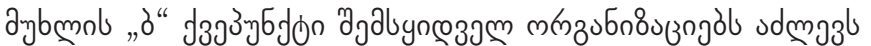

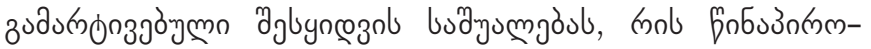

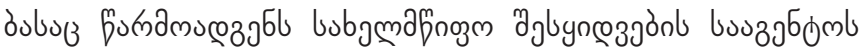

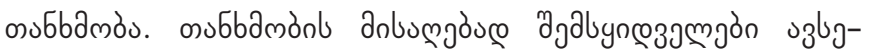

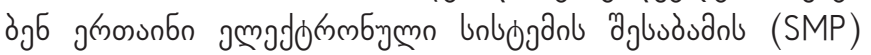

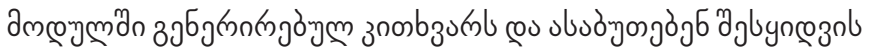

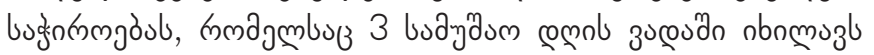

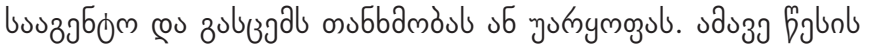

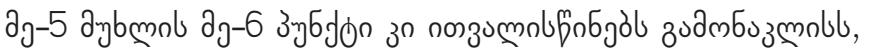

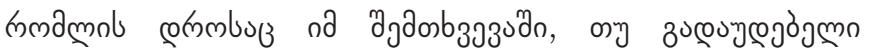

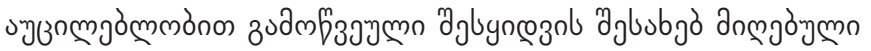

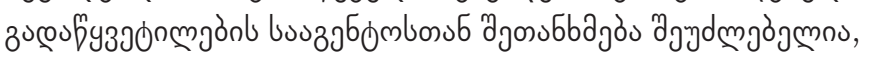
kuœ

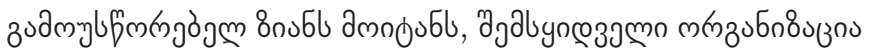

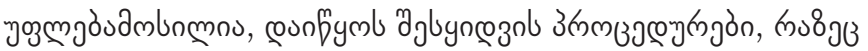

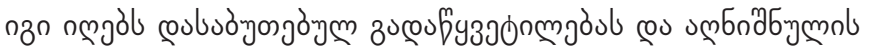

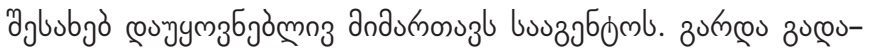

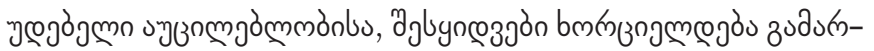

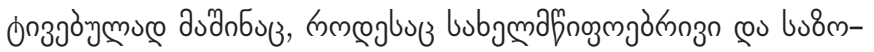

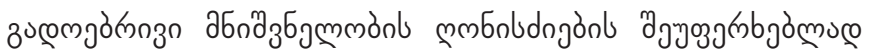

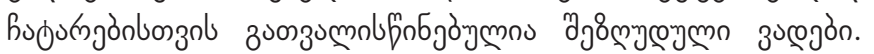

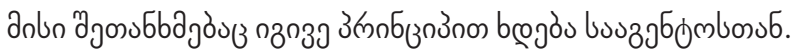

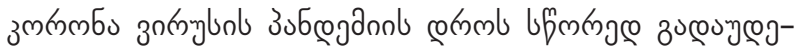

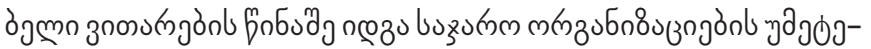

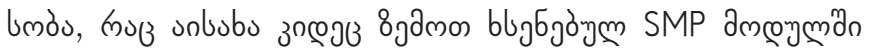

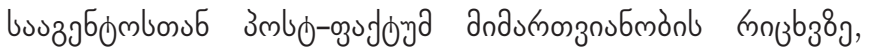

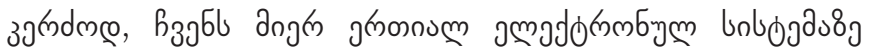

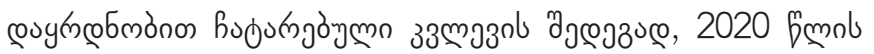

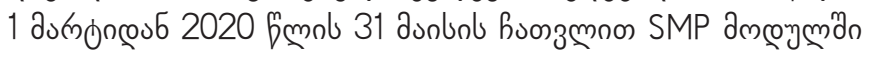

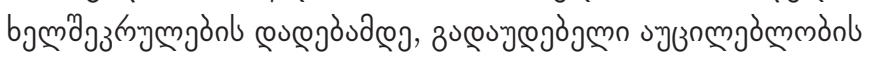

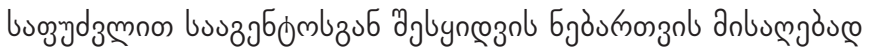

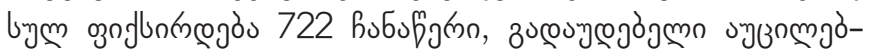

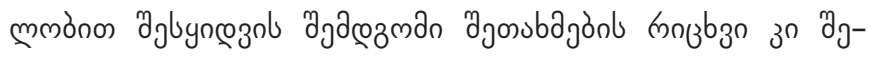

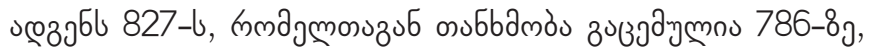

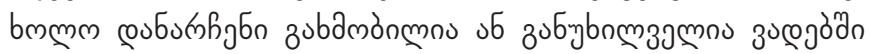

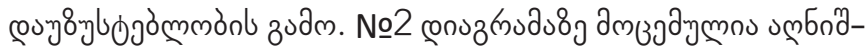

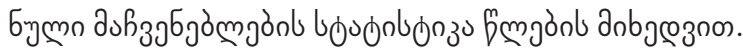

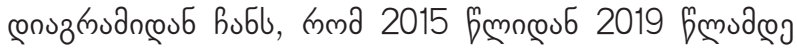

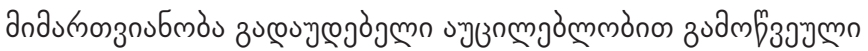

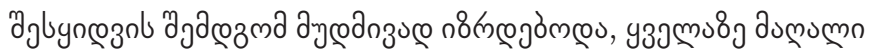

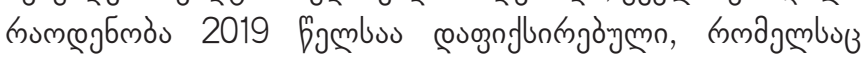

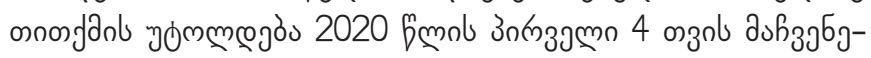

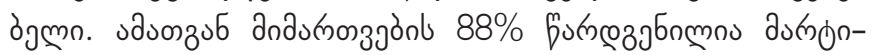

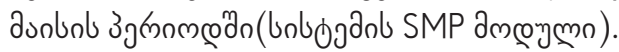

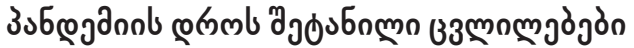

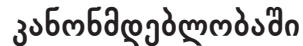

2020 f̧mol 1 aunonn

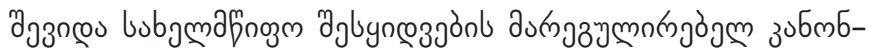

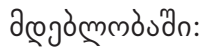

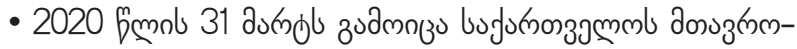

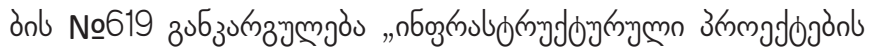

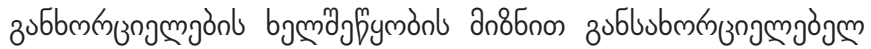

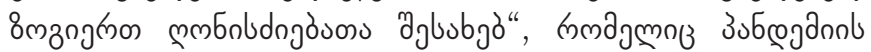

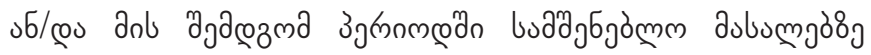

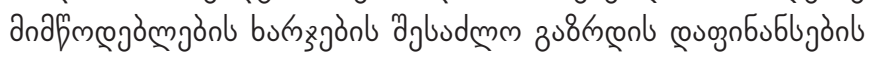

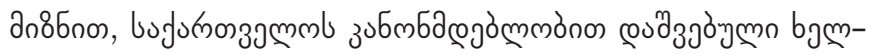

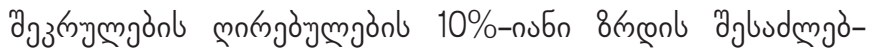

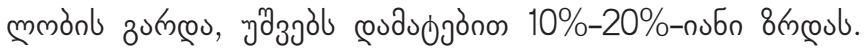

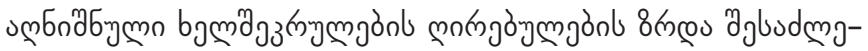

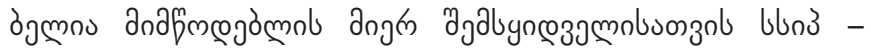

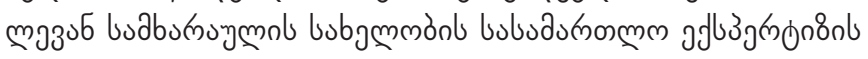

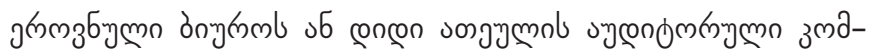

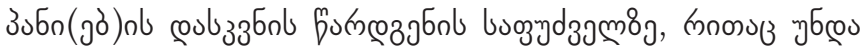

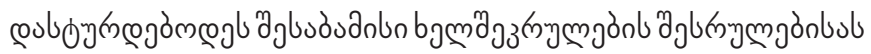

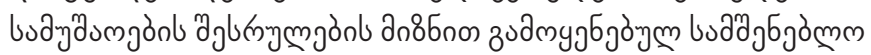

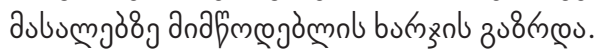




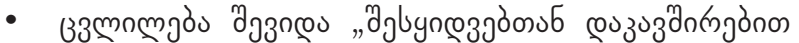

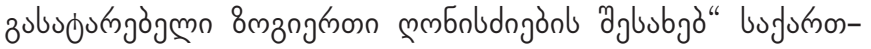

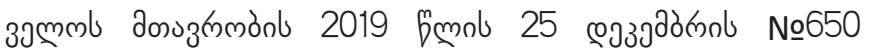

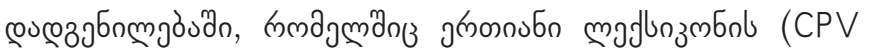

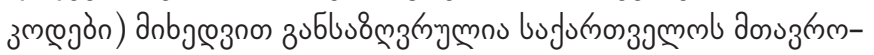

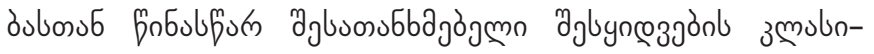

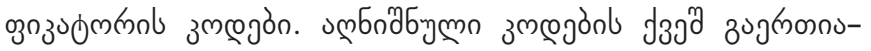

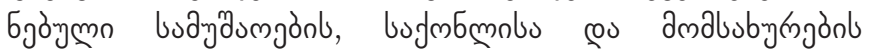

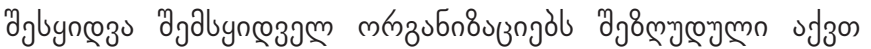

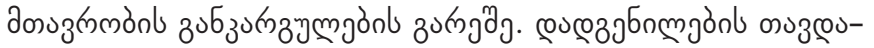

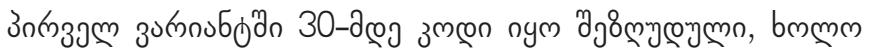

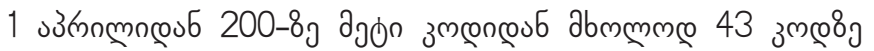

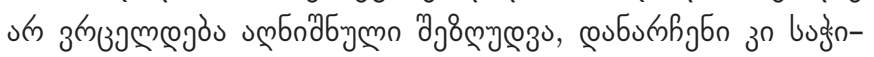

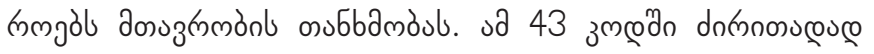

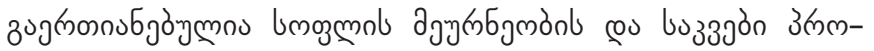

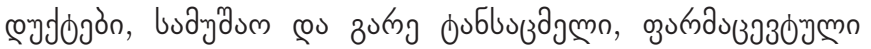

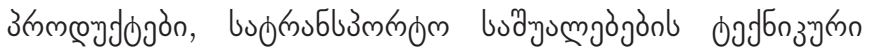

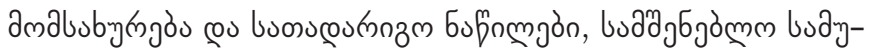

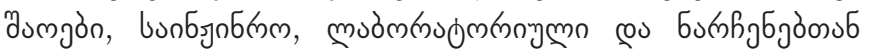

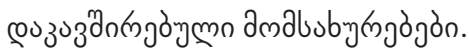

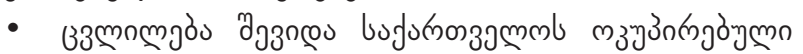

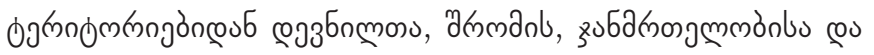

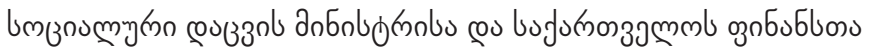

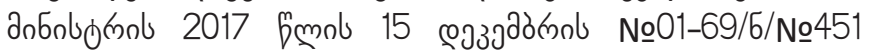

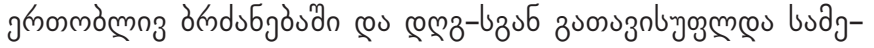

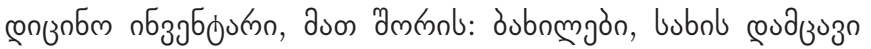

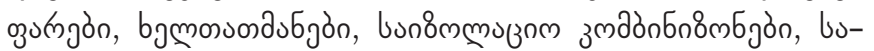

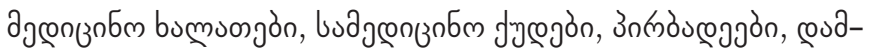

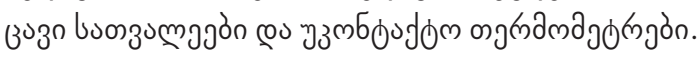

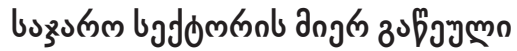

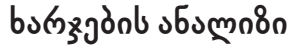

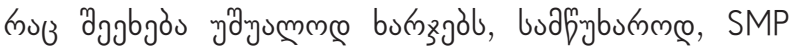

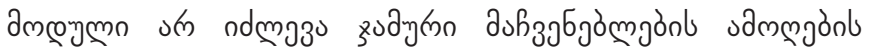

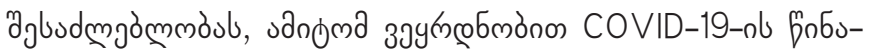

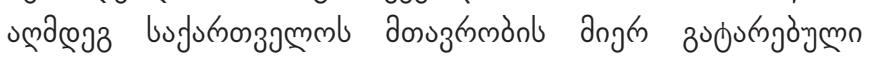

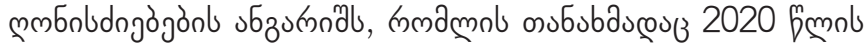

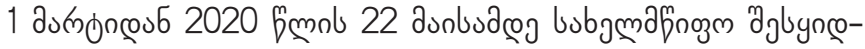

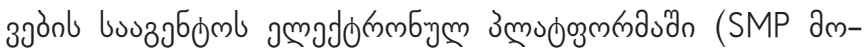

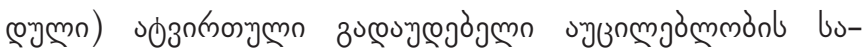

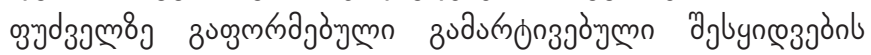

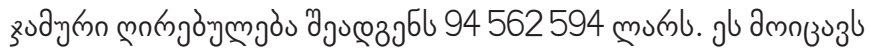

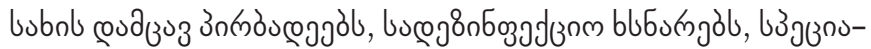

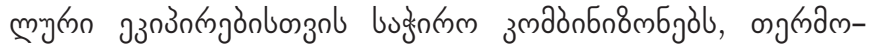

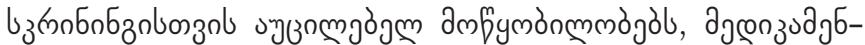

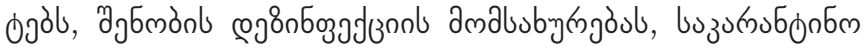

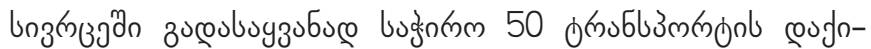

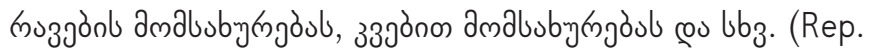
of Gov. 2020)

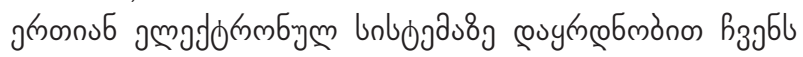

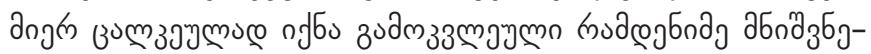

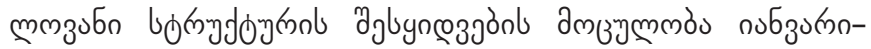

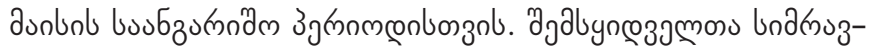

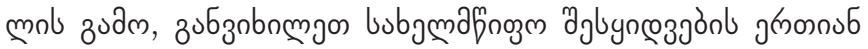

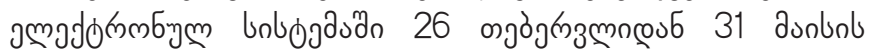

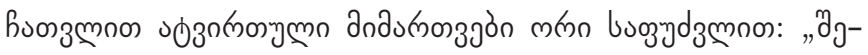

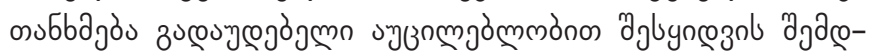

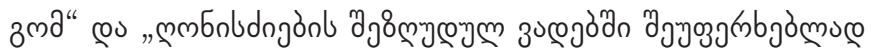

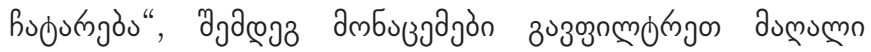

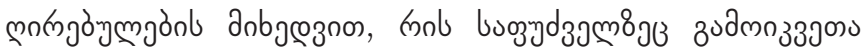
gдамэзо:

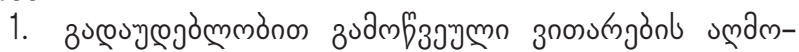

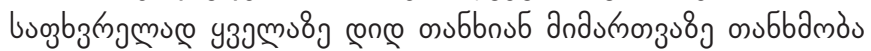

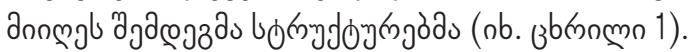

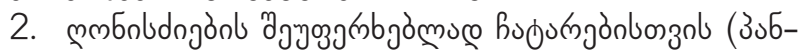

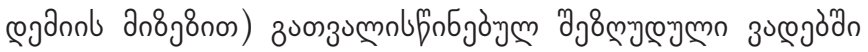

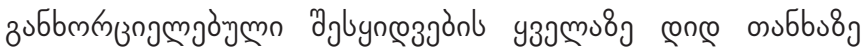

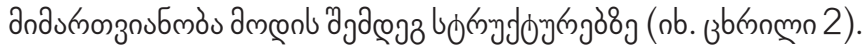

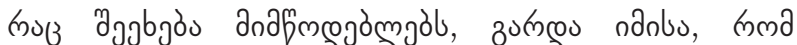

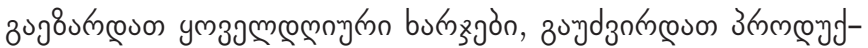

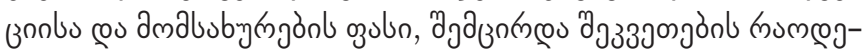

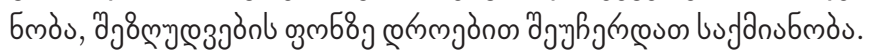

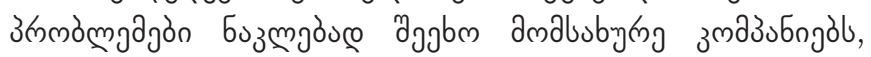

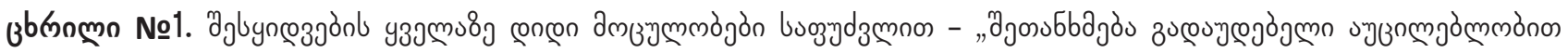

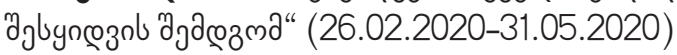

\begin{tabular}{|c|c|c|}
\hline 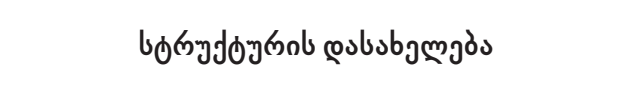 & 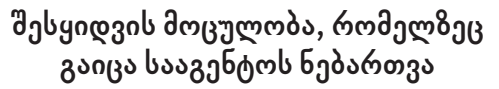 & 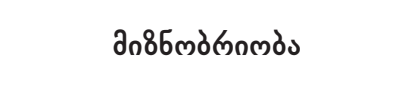 \\
\hline 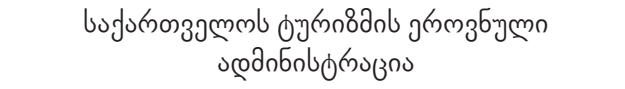 & 25975674.96 mußno & 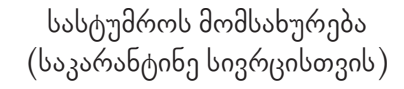 \\
\hline 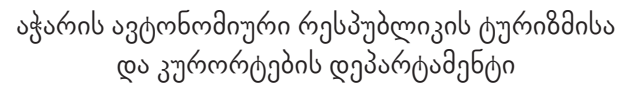 & 4066039 motro & 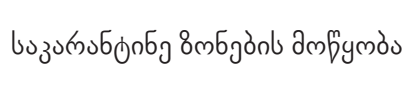 \\
\hline 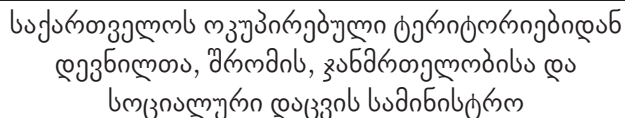 & 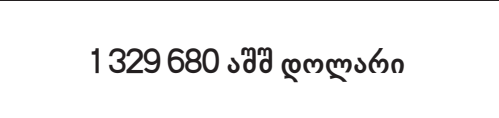 & 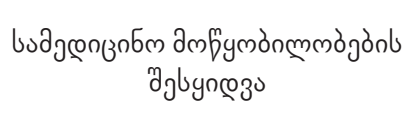 \\
\hline
\end{tabular}




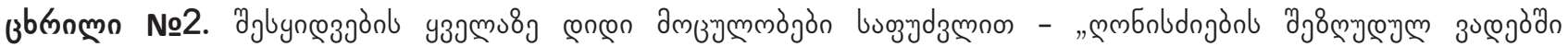

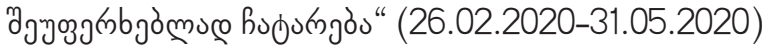

\begin{tabular}{|c|c|c|}
\hline 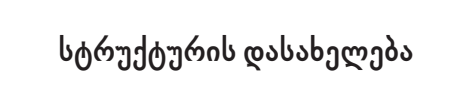 & 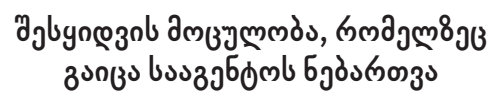 & д०86m \\
\hline 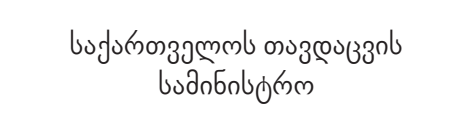 & 9500000 mుకno & 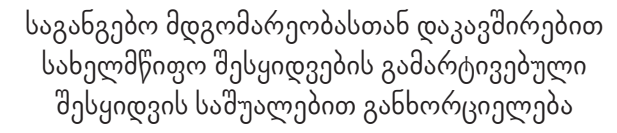 \\
\hline 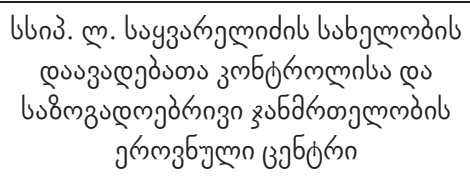 & 5051556 mృ & 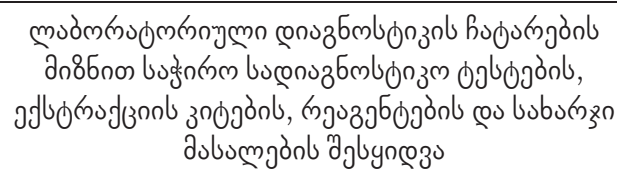 \\
\hline 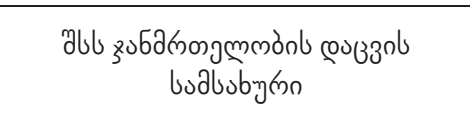 & 3000000 muKn & 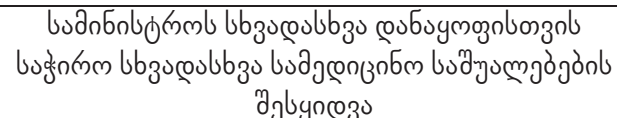 \\
\hline
\end{tabular}

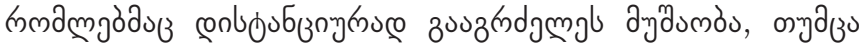

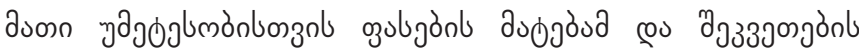

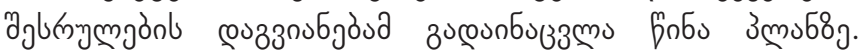

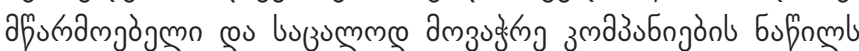

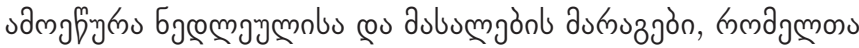

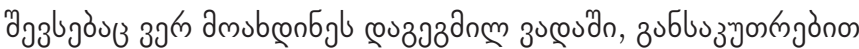

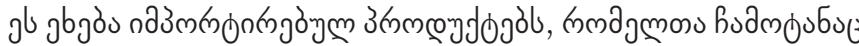

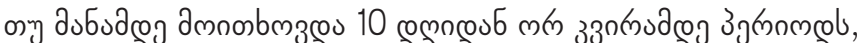

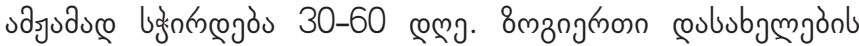

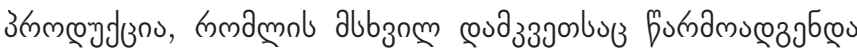

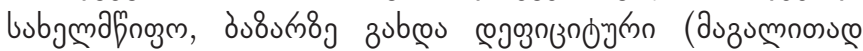

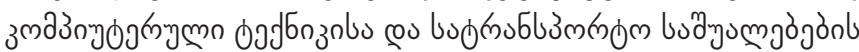

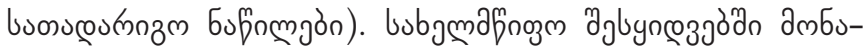

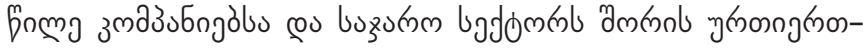

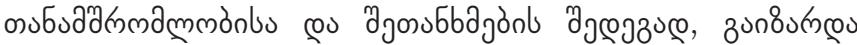

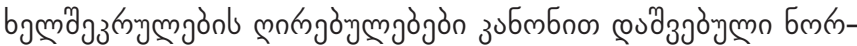

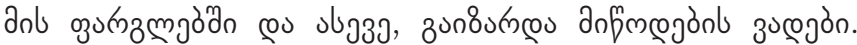

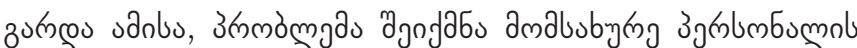

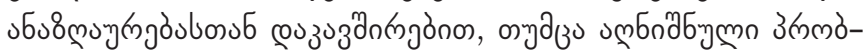

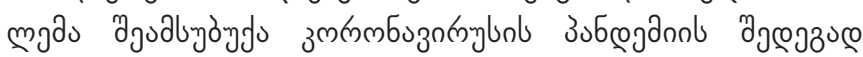

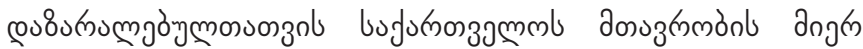

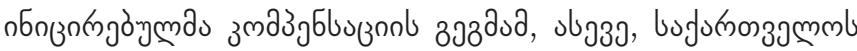

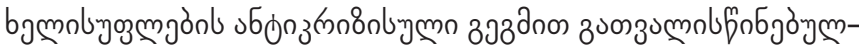

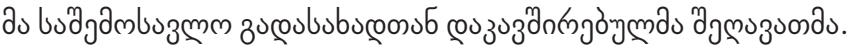

\section{@su335s}

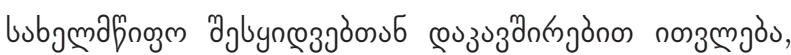

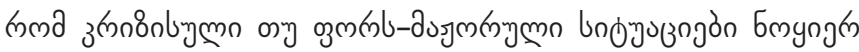

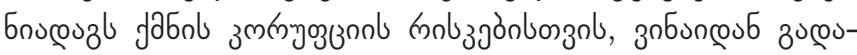

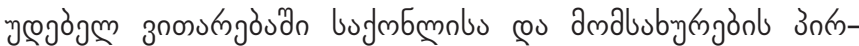

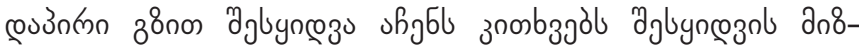

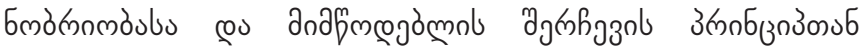

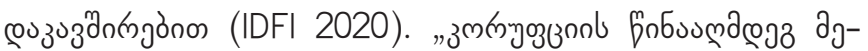

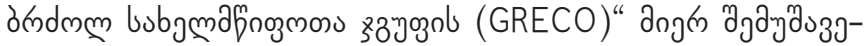

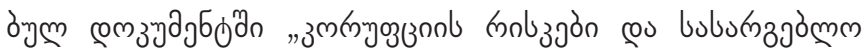

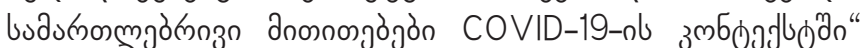

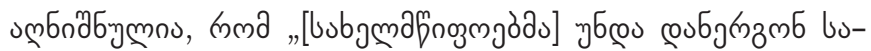

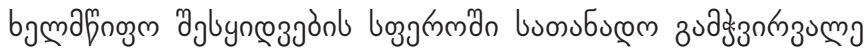

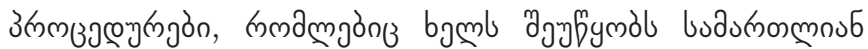

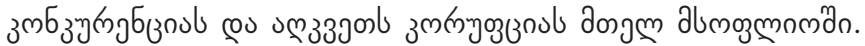

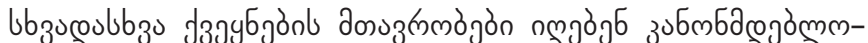

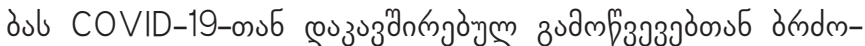

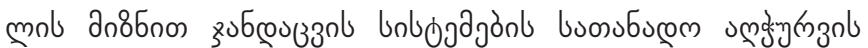

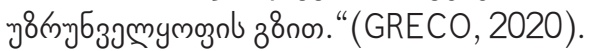

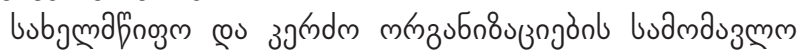

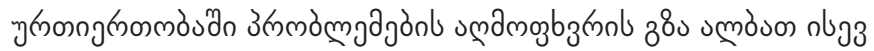

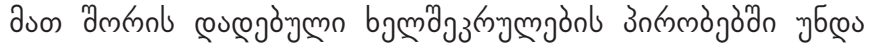

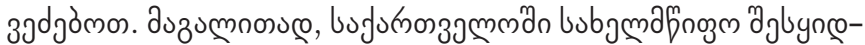

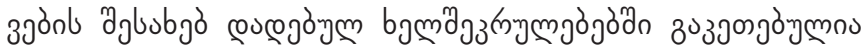

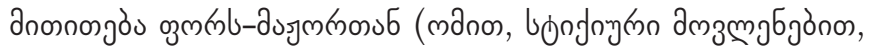

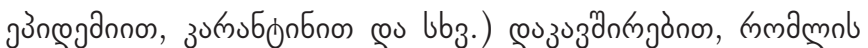

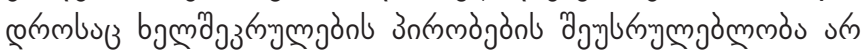

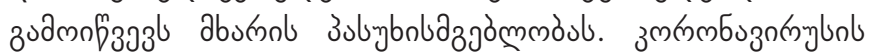

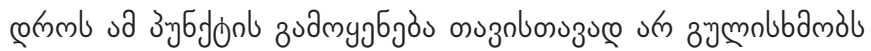

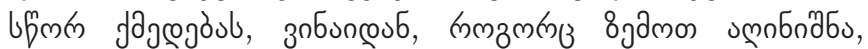

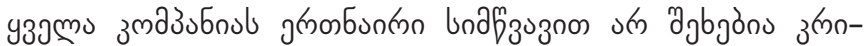

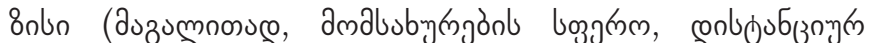

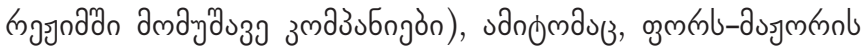

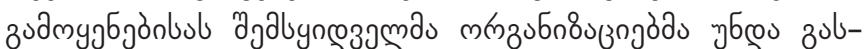

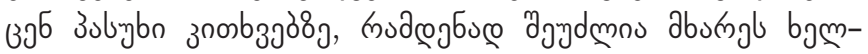

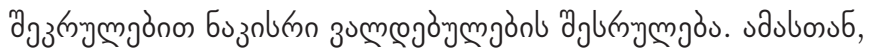

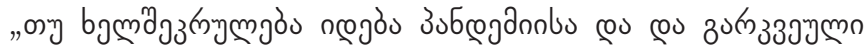

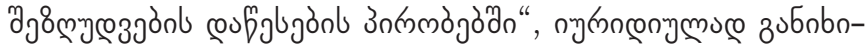

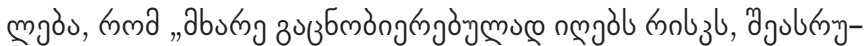

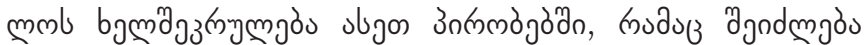

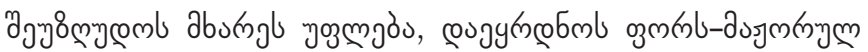
zurngamgoubl" (Nodia, Urumashvili and Partn.2020). 


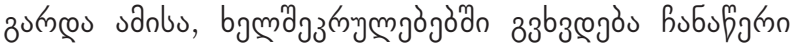

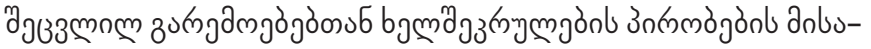

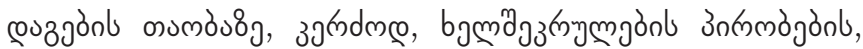

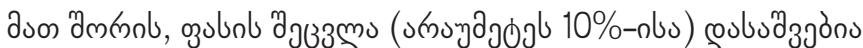

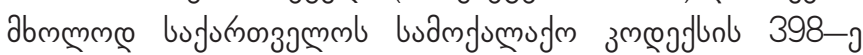

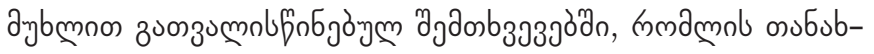

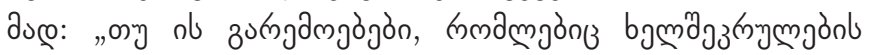

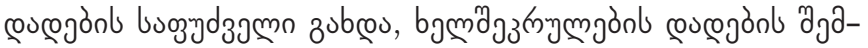

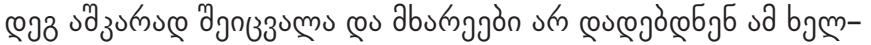

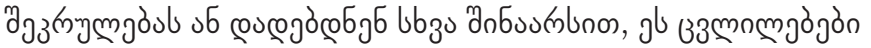

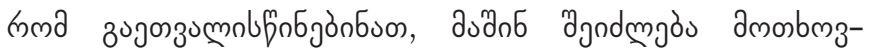

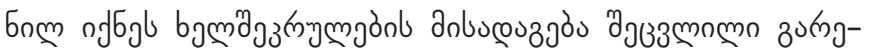

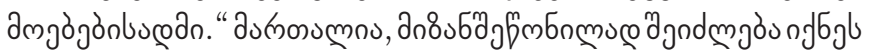

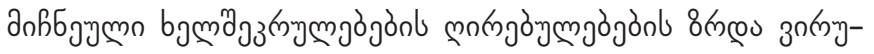

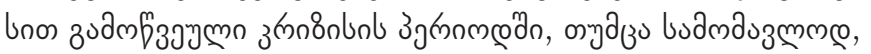

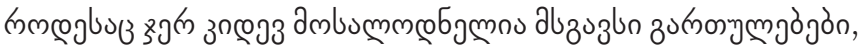

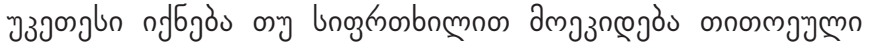

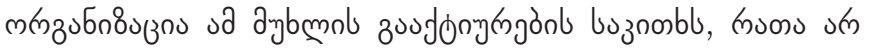

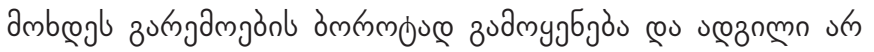

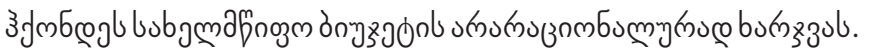

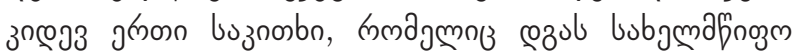

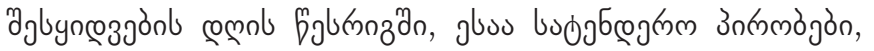

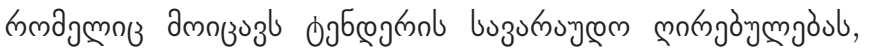

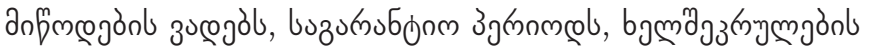

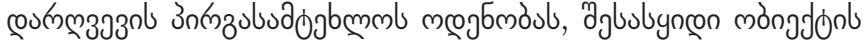

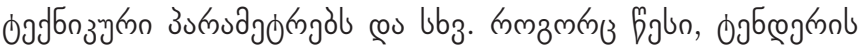

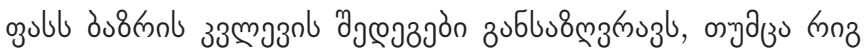

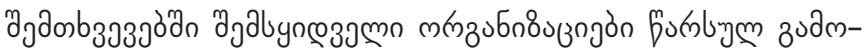

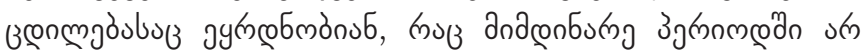

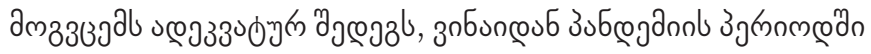

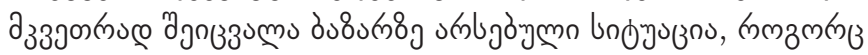

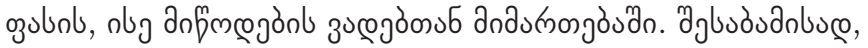

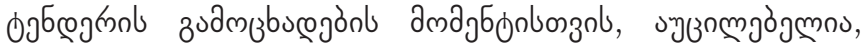

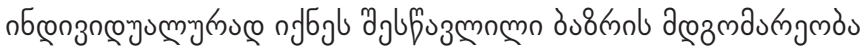

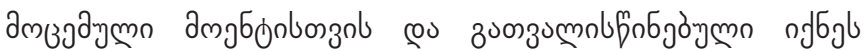

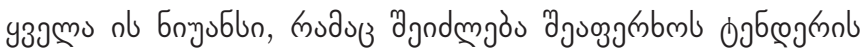

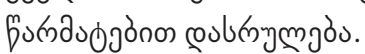

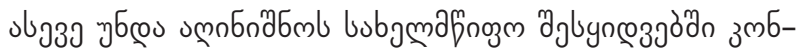

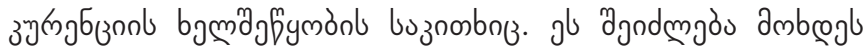

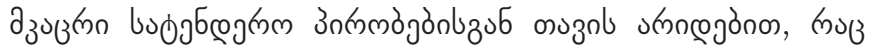

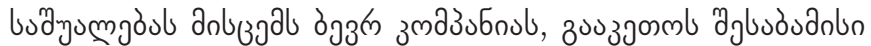

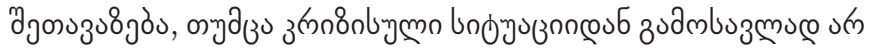

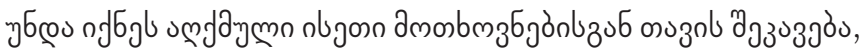

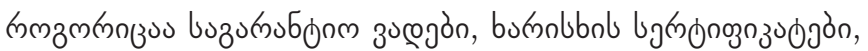

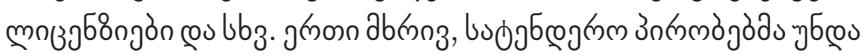

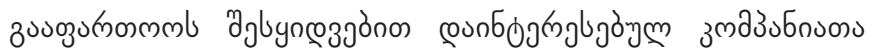

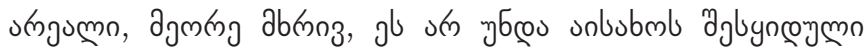
bufmbmalus on amabsumgngonol bumnlbbog.

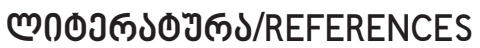

Babichi I., Keshelava D. \& Mzhavanadze G., (2020). «Economic Response to COVID-19: How does Georgia cope with the challenge?», ISET, Tbilisi, in Georgian.

Duncker K., Aarikka R. \& Puisto A., (2020) „Impacts of COVID-19 on public procurement in the EU“, Bird\&Bird's. https://www. twobirds.com/en/news/articles/2020/finland/koronaviruksen-vaikutukset-julkisiin-hankintoihin.

Emch A. \& Lyu Q., (2020). Reforms for China's public procurement regime expected in 2020. https://www.hlregulation. com/2020/03/18/reforms-for-chinas-public-procurement-regime-expected-in-2020/

Hausmann, R., (2020). Flattening the COVID-19 Curve in Developing Countries. Project Syndicate.https://www.projectsyndicate.org/commentary/flattening-covid19-curve-in-developing-countries-by-ricardo-hausmann-2020-03?referral= 208627\&barrier=accesspaylog

Khutsishvili, N., (2019). Economic Policy Stimulating Private Entrepreneurship, Tbilisi. (In Georgian)

Kikvadze, O., (2016). Improving Public Procurement in Public Financial Administration, Tbilisi. (In Georgian)

Krugman, P., (2020). We're not ready for a pandemic, The New York Times. https://messaging-custom-newsletters.nytimes. com/template/oakv2?uri=nyt://newsletter/87c61d7b-b428-4535-a94b-212a5a6090e5\&productCode=PK\&te=1\&nl=pa ul-krugman\&emc=edit_pk_20200225\&campaign_id=116\&instance_id=16271\&segment_id=21604\&user_id=bcb6ec40c a81f55384d37740da6f6b46\&regi_id=76197941edit_pk_20200225

Nadaraia, O., Chachanidze M., Bluashvili A., Kordzaia T., (2020). Impact of Covid19 on the business environment, TBC Capital, Tbilisi. (In Georgian)

Nodia, Urumashvili \&Partners (2020). Legal Review of the State of Emergency, Tbilisi. (In Georgian)

Papava V., (2020). Coronomic and Economic in the Face of Crisis, Tbilisi. (In Georgian) https://for.ge/view/182671/koronomikada-ekonomika-krizisis-winaSe.html .

Roubini N., (2020). A Greater Depression? Project Syndicate. https://www.project-syndicate.org/commentary/coronavirusgreater-great-depression-by-nouriel-roubini-2020-03?utm_source=Project\%20Syndicate\%20Newsletter\&utm_ campaign=80fd5aabf4-sunday_newsletter_29_03_2020\&utm_medium=email\&utm_term=0_73bad5b7d8-80fd5aabf493567601\&mc_cid=80fd5aabf4\&mc_eid=e9fb6cbcc0\&barrier=accesspaylog. 
Steingrüber, S., Kirya, M., Jackson, D., \& Mullard, S., (2020). Corruption in the time of COVID-19: A double-threat for low income countries.

https://www.u4.no/publications/corruption-in-the-time-of-covid-19-a-double-threat-for-low-income-countries.

Chairman of the State Procurement Agency (2015). Approval the rules for defining simplified procurement criteria and simplified procurement, Order 13, Tbilisi, in Georgian.

http://procurement.gov.ge/getattachment/ELibrary/LegalActs/gamartivebuli_Sesyidvis_Catarebis_wesi.pdf.aspx

GRECO (2020), Risks of Corruption and Useful Legal Guidelines in the Context of COVID-19, Tbilisi, in Georgian. https://rm.coe. int/georgian-version-corruption-covid-19/16809e3bf4.

IDFI (2020). Analysis of the bureaucratic expenses of the emergency budget. Tbilisi. (In Georgian)

https://idfi.ge/public/upload/Covid/analysis_of_the_bureaucratic_expenses_of_the_emergency_budget.pdf.

IDFI (2020). Guidelines on the Proactive Publication of Information by Governments during the Covid-19 Crisis and on Covid-19 Related Public Procurement. Tbilisi.

https://idfi.ge/ge/guidelines_on_the_proactive_publication_of_information_by_governments_during_the_covid-19_crisis_ and_on_covid-19_related_public_procurement.

Legislative Herald of Georgia, Decree of the Government of Georgia №619, Some Measures to be Implemented to Support the Implementation of Infrastructure Projects, Tbilisi. (In Georgian)

https://matsne.gov.ge/ka/document/view/4843736?publication=0

Legislative Herald of Georgia (2017). Order of Minister of Labour, Health, and Social Affairs of Georgia 01-69/451, Determining the list of goods intended for medical / medical purposes, the supply and / or import of which is exempt from value added tax, Tbilisi. (In Georgian)

https://www.matsne.gov.ge/ka/document/view/3893311?publication=3

Legislative Herald of Georgia, Civil Code of Georgia, Article 398, Tbilisi. (In Georgian)

https://matsne.gov.ge/ka/document/view/31702?publication=109

State procurement agency (SPA), Annual report 2019. Tbilisi.

http://procurement.gov.ge/getattachment/ELibrary/AnalyticalStudiesReports/Angarishi_2019_GEO.pdf.aspx

Report of Government of Georgia against COVID-19 (2020). Tbilisi. (In Georgian)

http://gov.ge/files/76338_76338_444796_COVID-19angarishi...pdf 